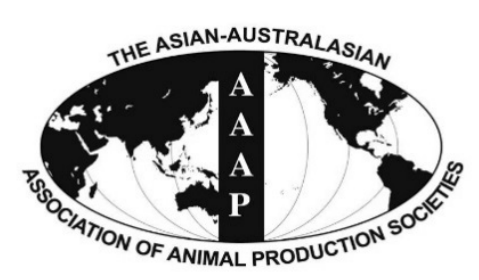

Open Access

Asian Australas. J. Anim. Sci.

Vol. 29, No. 10 : 1508-1514 October 2016

http://dx.doi.org/10.5713/ajas.15.0892

www.ajas.info

pISSN 1011-2367 elSSN 1976-5517

\title{
Preparation and Characterization of an Antibody Antagonist That Targets the Porcine Growth Hormone Receptor
}

\author{
Huanzhong Cui, Yanrong Wang ${ }^{1}$, Meng Song, and Hui Zhang* \\ College of Animal Science and Technology Jilin Agricultural University, Changchun 130118, China
}

\begin{abstract}
A series of antagonists specifically targeting growth hormone receptors (GHR) in different species, such as humans, rats, bovines, and mice, have been designed; however, there are currently no antagonists that target the porcine growth hormone (GH). Therefore, in this study, we developed and characterized a porcine GHR (pGHR) antibody antagonist (denoted by AN98) via the hybridoma technique. The results from enzyme-linked immunosorbent assay, fluorescence activated cell sorter, indirect immunoinfluscent assay, and competitive receptor binding analysis showed that AN98 could specifically recognize pGHR, and further experiments indicated that AN98 could effectively inhibit pGH-induced signalling in CHO-pGHR cells and porcine hepatocytes. In addition, AN98 also inhibited GH-induced insulin-like growth factor-1 (IGF-1) secretion in porcine hepatocytes. In summary, these findings indicated that AN98, as a pGHR-specific antagonist, has potential applications in pGH-pGHR-related research on domestic pigs. (Key Words: Porcine Growth Hormone, Porcine Growth Hormone Receptors, Signalling Pathway, Antagonist)
\end{abstract}

\section{INTRODUCTION}

The growth hormone (GH) is a 191 amino acid single chain protein that is mainly synthesized and released in the pituitary gland (Abdel-Meguid et al., 1987). GH displays physiological effects in growth, development, and metabolism. The physiological actions of GH are mediated by the growth hormone receptor (GHR), which is expressed on target cells and tissues. After the GH binds to the GHR, janus kinase 2 (JAK2) is activated, and this activates downstream signalling molecules, such as the signal transducer and activator of transcription 5/3/1(STAT5/3/1), phosphatidylinositol 3-kinase (PI 3-kinase)/ serine/threonine kinase (AKT) and extracellular regulated protein kinases (ERK1/2). These activated signalling molecules translocate into the nucleus, where they regulate the transcription of targeted genes (Brooks and Waters, 2010; Brooks et al., 2014).

\footnotetext{
* Corresponding Author: Hui Zhang. Tel: +86-0431-84533462, Fax: +86-0431-84533462, E-mail: chzh@jlau.edu.cn

${ }^{1}$ Henan University of Science and Technology, Xinxiang, Henan 471023, China.

Submitted Oct. 28, 2015; Revised Nov. 22, 2015; Accepted Nov. 30, 2015
}

The role of the porcine GH (pGH) in domestic pigs has been extensively studied, which has revealed that pGH could initiate physiological effects, such as the promotion of pig growth, foetus development, adipose tissue accretion and metabolism, and numerous other physiological functions (Chung et al., 1985; Gatford et al., 2004; Ramsay et al., 2005). However, many physiological functions of pGH have not been identified. Therefore, it is important to develop an antagonist for the porcine GHR (pGHR), which can be used to determine the underlying biological functions of the pGH-pGHR system, and for other pGHRrelated studies.

The antagonists targeting the GHRs of different species, such as humans (van der Lely et al., 2001), rats (Lan et al., 2015b), and bovine (Chen et al., 1991) have been investigated. However, there are no current studies investigating antagonists specifically targeting the pGH. In addition, it is unclear whether the antagonists targeting GHR in other species can recognize pGHR. Therefore, in this study, we developed and characterized a pGHR-specific antibody antagonist (denoted by AN98), and found that AN98 effectively inhibited pGH signalling in vitro. Furthermore, AN98 also inhibited pGH binding to pGHR, 
which suggests there are common epitopes shared by AN98 and pGH. In addition, AN98 inhibited the secretion of insulin-like growth factor (IGF-1) by pGH-induced porcine hepatocytes. In summary, current observations indicated that AN98, a pGHR-specific antagonist, should be considered when investigating the roles of pGH and pGHR in domestic pigs.

\section{MATERIALS AND METHODS}

\section{Antibodies, reagents, and cells}

JAK2 and phospho-JAK2, signal transduction and activator of transcription (STAT5) and phospho-STAT5 antibodies were obtained from Santa Cruz Biotechnology, Inc. (Santa Cruz, CA, USA). Horseradish peroxidase (HRP)-conjugated goat anti-rabbit and anti-mouse antibodies were obtained from Sigma-Aldrich (SigmaAldrich Company, St. Louis, MO, USA). The IP kit, enhanced chemiluminescent (ECL), and Western Blotting Detection Kit were obtained from Pierce (Puyallup, WA, USA). The cell lysis buffer and BCA Protein Assay Kit were obtained from Thermo Fisher Scientific (Waltham, MA, USA). The extracellular domain of rat growth hormone binding protein (GHBP), human GHBP and porcine GHBP was provided by HuaCheng (Changchun, China). The porcine IGF-1 enzyme-linked immunosorbent assay (ELISA) kit was purchased from Hua Yi Medical and Biological Laboratories Co., Ltd (Changchun, China). All reagents were from Sigma-Aldrich unless otherwise stated.

Porcine hepatocytes and $\mathrm{CHO}$ cells transfected with pGHR were cultured according to our published methods (Lan et al., 2015a).

\section{Preparation of the anti-pGHR monoclonal antibody}

Anti-pGHR monoclonal antibodies were prepared using the hybridoma technique as previously described (Lan et al., 2014). Six- to eight-week-old BALB/c mice were immunized with $0.3 \mathrm{mg}$ porcine GHBP emulsified in Freund's complete adjuvant. The animals received four similar booster injections of GHBP at 14-day intervals. After a series of immunizations, all mice generated high titres of antibodies against porcine GHBP, which was determined by ELISA. Three days after the final immunization, the splenocytes from immunized mice were fused with $\mathrm{Sp} 2 / 0$ myeloma cells using a 50\% PEG1500 solution. After fusion, the cells were distributed in 96-well cell culture plates at $1 \times 10^{5}$ cells/well; hybridomas were cultured in HAT medium. ELISAs were performed to demonstrate the presence of antibodies against the porcine GHBP.

\section{Enzyme-linked immunosorbent assay}

Indirect ELISAs were conducted to detect monoclonal antibodies against pGHBP as described as previously (Lan et al., 2015a). ELISA plates were coated with pGHBP (100 $\mu \mathrm{L} /$ well) for $12 \mathrm{~h}$ at $4^{\circ} \mathrm{C}$. After washing three times with phosphate buffer solution with tween-20 (PBST), the plates were blocked with 3\% bovine serum albumin (BSA) for $2 \mathrm{~h}$ $37^{\circ} \mathrm{C}$. Then, hybridoma supernatant was added to the plates, and incubated for $12 \mathrm{~h}$ at $4^{\circ} \mathrm{C}$. The plates were then washed three times, and HRP-conjugated goat anti-mouse IgG secondary antibody was added and incubated for $2 \mathrm{~h}$ at room temperature. After three washes with PBST, the plates were introduced to a colorimetric reaction consisting of a 3,3',5,5'-tetramethylbenzidine (TMB) substrate solution and incubated for $15 \mathrm{~min}$ at room temperature. Final measurements were taken with an automatic ELISA plate reader (Multiskan FC, Thermo Fisher Scientific Inc., USA).

\section{Fluorescence activated cell sorter analysis}

CHO-pGHR cells were cultured for $12 \mathrm{~h}$ in serum-free medium, and then at least 50,000 cells were collected and incubated with AN98 or an isotype control for $0.5 \mathrm{~h}$ at $4{ }^{\circ} \mathrm{C}$. After washing with phosphate buffer solution (PBS), the cells were incubated with a Fluorescein isothiocyanate (FITC)-conjugated secondary antibody (1:200 dilution) for $1 \mathrm{~h}$ in the dark at $4^{\circ} \mathrm{C}$. After washing steps, the labelled cells were analysed using a FACSCalibur flow cytometry device (Becton Dickenson, Franklin, NJ, USA). Data were analysed using Cell Quest software.

\section{Indirect immunofluorescence}

Porcine hepatocytes grown on collagen-coated glass coverslips were fixed with $4 \%$ paraformaldehyde at $37^{\circ} \mathrm{C}$ for $0.5 \mathrm{~h}$. After washing the coverslips three times with PBS, the cells were blocked with $1 \%$ BSA for $1 \mathrm{~h}$. After three washes with PBS, the cells were incubated with AN98 or an isotype control monoclonal antibody (negative control) for $0.5 \mathrm{~h}$ at $37^{\circ} \mathrm{C}$. Cells were then incubated with FITC-labelled goat anti-mouse IgG secondary antibody (1:200 dilutions) for $0.5 \mathrm{~h}$ at $37^{\circ} \mathrm{C}$ in the dark. The cells were washed again and incubated with Hoechst for $15 \mathrm{~min}$ at $37^{\circ} \mathrm{C}$. After washing, cells were analysed using confocal laser scanning microscopy (Olympus FV1000, Tokyo, Japan).

\section{Competitive receptor-binding assay}

Competitive receptor-binding assays were conducted to evaluate if AN98 competed with pGH binding to pGHR. Before experiments, CHO-pGHR cells were cultured in serum-free media containing 1\% BSA for $12 \mathrm{~h}$. The cells were then collected, pipetted into FACS tubes $\left(2 \times 10^{5}\right.$ cells/tube), and incubated with FITC-pGH and different concentrations of unlabelled pGH, AN98 or an isotype control for $1 \mathrm{~h}$ in the dark at $4^{\circ} \mathrm{C}$. After three washes with PBS, labelled cells were analysed on a FACSCalibur Flow Cytometer (Becton Dickenson, USA). Data were analysed 
using Cell Quest software.

\section{Western blot analysis}

The antagonistic effects of AN98 were tested with western blot analysis, which was performed as previously described (Lan et al., 2014). Briefly, the CHO-pGHR cells or porcine hepatocytes were cultured in serum starvation media for $10 \mathrm{~h}$. A constant amount of pGH (100 ng) was pre-incubated with different concentrations of AN98, and then, the mixtures were added to the plates for $25 \mathrm{~min}$ at $37^{\circ} \mathrm{C}$. After stimulations, the cells were solubilized in lysis buffer and incubated for $30 \mathrm{~min}$ on ice. Cell debris was removed with centrifugation. The resulting supernatants were harvested and subjected to ultrafiltration. Protein concentration was detected with the Bio-Rad protein assay (Bio-Rad Company, Osaka, Japan). Samples were first boiled for $5 \mathrm{~min}$ in $1 \times \mathrm{SDS}$, then protein samples $(30 \mu \mathrm{g}$ each lane) were subjected to sodium dodecyl sulfatepolyacrylamide gel electrophoresis (SDS-PAGE) and transferred to a polyvinylidene fluoride (PVDF) membrane. After blocking the membrane with 5\% non-fat milk, the membrane was probed with phospho-JAK2 phosphoSTAT5 antibodies according to the manufacturer's protocols. The membranes were then washed and incubated with a secondary antibody conjugated to HRP for $2 \mathrm{~h}$. After three washes with PBST, protein bands were detected through the ECL system.

Measurement of insulin-like growth factors-1 content in

\section{the medium}

Freshly isolated hepatocytes $\left(5.0 \times 10^{6}\right)$ were seeded into $\mathrm{T}-100$ flasks that were pre-coated with pigtail collagen for 5 h. After cell attachment, the plates were washed with PBS and cells were treated with serum-free medium for $8 \mathrm{~h}$. Then, the cells were stimulated with $20 \mathrm{nM}$ pGH alone (positive control) or with $20 \mathrm{nM}$ pGH together with increasing concentrations of AN98 for $12 \mathrm{~h}$. After incubation, the medium was harvest. The IGF-1 content from each experimental condition was determined using the porcine IGF-1 ELISA kit.

\section{Statistical analysis}

Data are presented as the mean values \pm standard error.

\section{RESULTS}

\section{Generation and characterization of anti-pGHR monoclonal antibody}

We characterized an anti-pGHR antibody (denoted by AN98) using the hybridoma technique. A series of experiments were used to characterize this antibody. First, the ELISA results indicated that AN98, but not the control antibody, specifically react with the porcine GHBP (Figure 1A). Second, to further check the specificity of AN98, the GHBPs from different species (human, rat, sheep, and bovine) were coated on ELISA plates. The corresponding ELISA assays illustrated that AN98 specifically reacted with porcine GHBP but not the GHBPs from other species
A

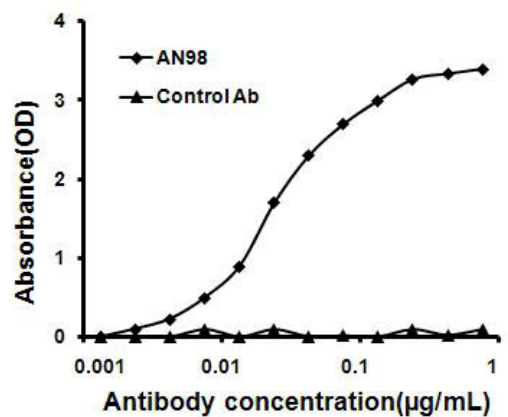

C
B

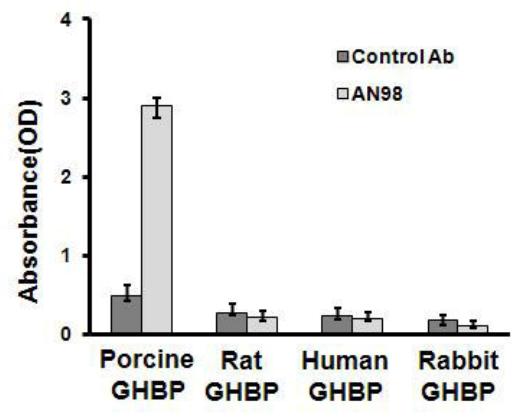

Figure 1. AN98 specifically recognized porcine GHBP. (A) AN98 reacted with porcine GHBP. ELISA plates were coated with porcine GHBP, after blocking and washing, different concentrations of AN98 or the control antibody were added, and followed the treatments described in the materials and methods. (B) AN98 specifically recognized porcine GHBP in ELISA assays. ELISA plates were coated with GHBP from different species. After blocking and washing, the AN98 and the control antibody were added. (C) AN98 specifically recognized porcine GHBP, identified by western blot assays. The GHBPs from different species were separated by SDS-PAGE and transferred to a PVDF membrane, after blocking the membrane with 5\% non-fat milk, the membrane was probed with AN98 and the control antibody. The figures represent at least three independent experiments. GHBP, growth hormone binding protein; ELISA, enzymelinked immunosorbent assay; SDS-PAGE, sodium dodecyl sulfate-polyacrylamide gel electrophoresis; PVDF, polyvinylidene fluoride. 
(Figure 1B). Furthermore, the results from the western blot analysis also confirmed this (Figure 1C).

\section{AN98 specifically binds to pGHR on target cells}

The previously mentioned experiments indicated that AN98 specifically reacted with porcine GHBP. Next, we further identified the reaction of A98 with pGHR (fulllength pGHR) expressed on target cells. For this, we conducted the following three experiments: i) The results from FACS analysis illustrated that AN98 could bind to CHO-pGHR (Figure 2A). However, control antibody showed no positive signal; ii) Immunofluorescence (IFA) showed that AN98, but not the control antibody, bound to the CHO-pGHR (Figure 2B); iii) Competitive receptorbinding assays indicated that AN98 recognized pGHR expressed on target cells, and this experiment also indicated that the GHR binding epitopes of pGH overlap with that of AN98 (Figure 2C).

\section{Evaluation of the antagonistic effects of AN98}

Before evaluating the antagonistic effects of AN98 in CHO-pGHR cells, we first determined that AN98 itself has no agonistic effect. As shown in Figure 3, AN98 and the control antibody could not activate JAK2-STAT5 signalling, whereas $\mathrm{pGH}$ and $\mathrm{B} 32$ induced the phosphorylation of JAK2-STAT5. Next, we determined the AN98 antagonistic activities; AN98, but not the control antibody, effectively inhibited pGH-induced signalling. The antagonistic effects of AN98 were initially seen at a concentration of 0.05 $\mu \mathrm{g} / \mathrm{mL}$. The antagonistic effects of AN98 on JAK2-STAT5 were dramatically enhanced as the AN98 concentration increased. When a $5 \mu \mathrm{g} / \mathrm{mL}$ of AN98 was used, pGHinduced signalling was completely inhibited (Figure 4). These findings show that AN98 has antagonistic activities.

In addition, we checked the antagonistic effect of AN98 in porcine hepatocytes. As indicated in Figure 1, AN98, but not the control antibody, effectively inhibited pGH-induced signalling in porcine hepatocyte models. The antagonistic effect of AN98 was initially shown at concentrations of 0.05 $\mu \mathrm{g} / \mathrm{mL}$. The antagonistic effects of AN98 on JAK2-STAT5 signalling were dramatically augmented as the AN98 concentration increased. When AN98 was used at a concentration of $5 \mu \mathrm{g} / \mathrm{mL}$, pGH-induced signalling was completely disrupted. These findings demonstrated the
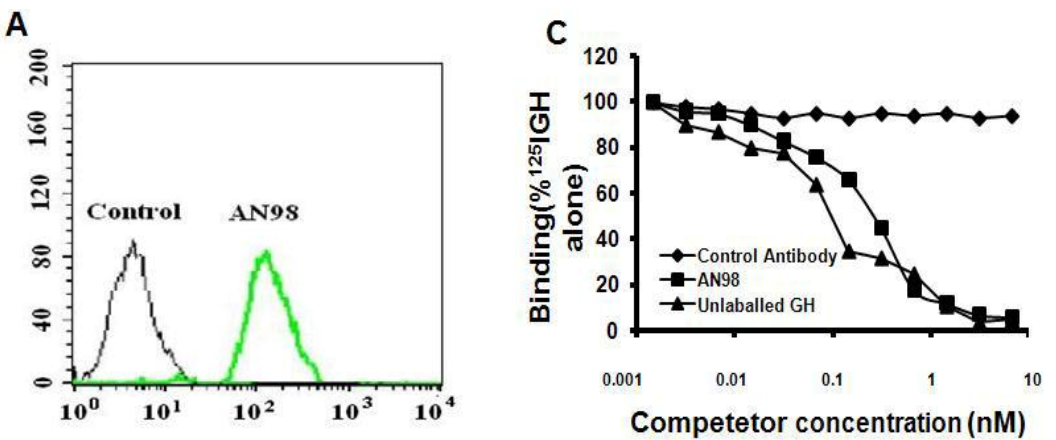

B
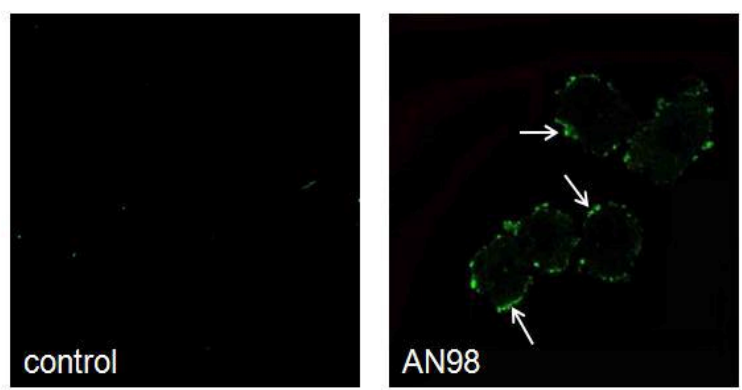

Figure 2. AN98 recognized full-length GHR. CHO-pGHR cells or hepatocytes were pre-treated as described in the materials and methods. (A) FACS analysis. CHO-pGHR cells were incubated with AN98 or an isotype control for $0.5 \mathrm{~h}$ at $4^{\circ} \mathrm{C}$. The cells were incubated with an FITC-conjugated secondary antibody. Labelled cells were analysed by a FACSCalibur flow cytometry device (Becton Dickenson, Franklin, NJ, USA). Data were analysed using Cell Quest software. (B) IFA analysis. Porcine hepatocytes were incubated with AN98 or an isotype control monoclonal antibody for $0.5 \mathrm{~h}$ at $37^{\circ} \mathrm{C}$. Cells were then incubated with FITC-labelled goat anti-mouse IgG secondary antibody for $0.5 \mathrm{~h}$ at $37^{\circ} \mathrm{C}$ in the dark. Cells were analysed with a confocal laser scanning microscopy (Olympus FV1000, Tokyo, Japan). (C) Competitive receptor-binding assays. CHO-pGHR cells were incubated with FITC-pGH and different concentrations of unlabelled pGH, AN98, or an isotype control for $1 \mathrm{~h}$ in the dark at $4^{\circ} \mathrm{C}$. After three washes with PBS, the labelled cells were analysed on a FACSCalibur Flow Cytometer (Becton Dickenson, USA). Data were analysed using Cell Quest software. GHR, growth hormone receptor; FACS, fluorescence activated cell sorter; FITC, fluorescein isothiocyanate; IFA, immunoinfluscent assay; PBS, phosphate buffer solution. 


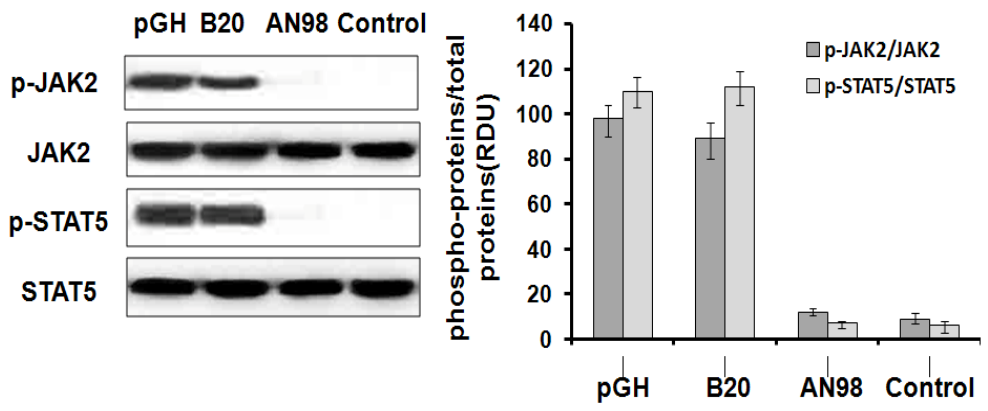

Figure 3. AN98 did not activate JAK2-STAT5. The CHO-PRLR cells were pre-treated as described in the materials and methods. The cells were treated with pGH, B32, AN98 or the control antibody $(20 \mathrm{nM})$ for $30 \mathrm{~min}$. Western blots were then performed with the indicated antibodies. The figures represent at least three independent experiments. JAK2-STAT5, janus kinase 2-signal transduction and activator of transcription.

antagonistic activities of AN98 in porcine hepatocytes.

\section{AN98 inhibits pGH-induced IGF-1 secretion in porcine hepatocytes}

To further test the antagonistic effects, we analysed the impact of AN98 on pGH-induced IGF-1 secretion in porcine hepatocytes. AN98 was shown to inhibit IGF-1 secretion induced by pGH. When AN98 was used at a concentration of $20 \mu \mathrm{g} / \mathrm{mL}$, almost all IGF-1 secretion was inhibited (Figure 6).

\section{DISCUSSION}

In this study, we prepared and characterized a monoclonal antibody against pGHR, denoted by AN98, which not only specifically binds to pGHR but also serves as a potential pGHR antagonist. This is the first report of a GHR antagonist that specifically targets pGHR.
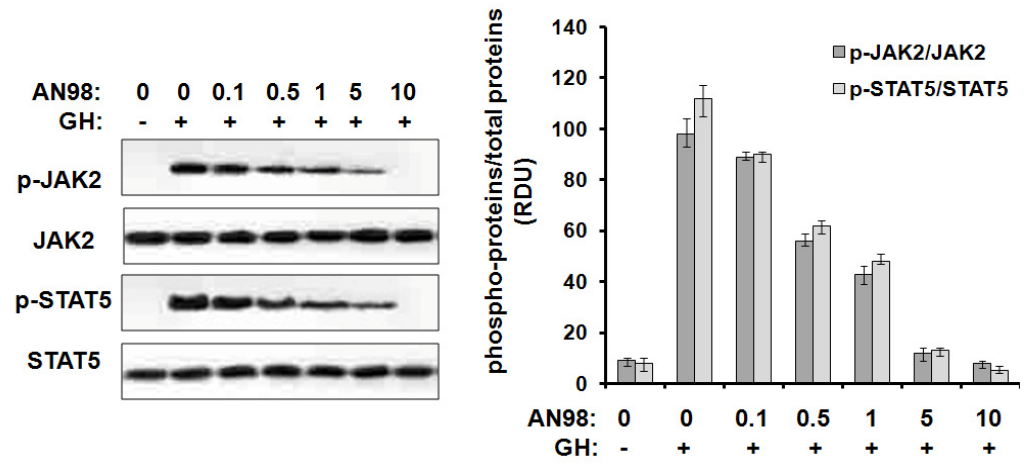

Figure 4. Inhibition of pGH-induced signaling by AN98 in CHO-GHR cells. The CHO-pGHR cells were cultured in serum starvation media for $10 \mathrm{~h}$. A constant amount of pGH (100 ng) was pre-incubated with different concentrations of AN98, then the mixtures were added to the plates for $25 \mathrm{~min}$ at $37^{\circ} \mathrm{C}$. After incubation, cell extracts were harvested, and samples $(30 \mu \mathrm{g}$ of protein per lane) were subjected to SDS-PAGE (10\% gel) and transferred to a PVDF membrane. After blocking the membrane with $5 \%$ non-fat milk, the membrane was probed with phospho-JAK2 phospho-STAT5 antibodies according to the manufacturer's protocols. The membrane was then washed and incubated with secondary antibody conjugated to HRP for $2 \mathrm{~h}$. After three washes with PBST, protein bands were detected using the ECL system. The figures represent at least three independent experiments. pGH, porcine growth hormone; GHR, growth hormone receptors; SDS-PAGE, sodium dodecyl sulfate-polyacrylamide gel electrophoresis; PVDF, polyvinylidene fluoride; JAK2, janus kinase 2; STAT5, signal transduction and activator of transcription; HRP, horseradish peroxidase; ECL, enhanced chemiluminescent. roles are displayed by the GHR. After GH binds to GHR, special conformation change(s) within the pre-dimerized ERK1/2, and AKT) are triggered (Brooks et al., 2014). Even though special conformational changes are required for GHR activation, the nature of this conformational change is still not fully understood. The Waters' group recently proposed a new model suggesting that the $\mathrm{GH}$ receptor exists predominantly as a dimer held together by its transmembrane helices and that GH binding to GHR induces a rotation of subunits that subsequently converts the ansmembrane helices into a left-hand crossover state, sing a repositioning of the intracellular domains (ICDs) activate JAK2 (Brooks et al., 2014). Structurally, the fulllength GHR consists of an extracellular domain (ECD), a single-pass transmembrane domain, and an ICD. The ECD 

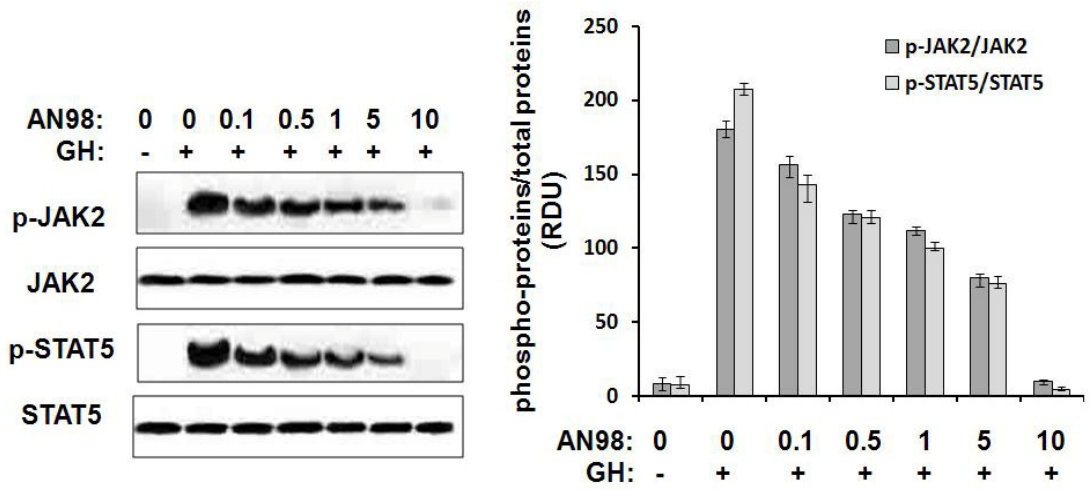

Figure 5. Inhibition of pGH-induced signalling by AN98 in porcine hepatocytes. Porcine hepatocytes were cultured in serum starvation media for $10 \mathrm{~h}$. A constant amount of pGH (100 ng) was pre-incubated with different concentrations of AN98, then the mixtures were added to the plates for $25 \mathrm{~min}$ at $37^{\circ} \mathrm{C}$. After incubation, cell extracts were harvested, and samples $(30 \mu \mathrm{g}$ of protein per lane) were subjected to SDS-PAGE (10\% gel) and transferred to a PVDF membrane. After blocking the membrane with 5\% non-fat milk, the membrane was probed with phospho-JAK2 phospho-STAT5 antibodies according to the manufacturer's protocols. The membranes were then washed and incubated with secondary antibody conjugated to HRP for $2 \mathrm{~h}$. After three washes with PBST, protein bands were detected using the ECL system. The figures represent at least three independent experiments. pGH, porcine growth hormone; SDSPAGE, sodium dodecyl sulfate-polyacrylamide gel electrophoresis; PVDF, polyvinylidene fluoride; JAK2, janus kinase 2; STAT5, signal transduction and activator of transcription; HRP, horseradish peroxidase; ECL, enhanced chemiluminescent; PBST, phosphate buffer solution with Tween-20.

(residues 1-246) of GHR consists of two subdomains (residues 1-123, referred to as subdomain 1; residues $128-$ 238, referred to as subdomain 2) (De Vos et al., 1992). Subdomain 1 of GHR-ECD is responsible for GH binding, and subdomain 2 is responsible for the conformational change(s) required for GHR activation. In the past few decades, several studies have reported GHR antagonists that target different species. GHR antagonists are divided into three classes consisting of the anti-GHR, GH analogue and anti-GH antibody: i) anti-GHR antagonist: Mab5 (a human GHR antibody antagonist) was prepared by Waters et al and was initially reported as a GHR antibody antagonist (Barnard et al., 1984). Similarly, another monoclonal antiGHR antibody (GF185), rat GHR antagonist, was characterized by Sun et al. (2015), along with its epitope, located in the receptor dimerization region of subdomain 1 on GHR-ECD; ii) GH analogue: G120R was developed by Kopchick and coworkers (Chen et al., 1994), their studies indicated that a single point mutation in $\mathrm{GH}$ at position 120 (glycine) converts GH from an agonist to an antagonist; iii) The anti-idiotypic antibody to GH: Lan et al. (2015b) recently proposed a new strategy for developing a GHR antagonist by preparing an anti-idiotypic antibody to $\mathrm{GH}$ (CG-86), which serves as a rat GHR antagonist. However, the above-mentioned studies reported GHR antagonists that target different species (such as rat, mouse, and human). Currently, there are no reports about pGHR-specific antagonists or whether the above-mentioned GHR antagonists targeting different species can recognize pGHR. Therefore, in this study, we developed a pGHR-specific antibody antagonist, AN98, which effectively inhibits GH- induced signaling (Figures 4 and 5), and inhibits IGF-1 secretion in primary hepatocytes.

A series of studies have indicated that the pGH-GHR system plays an important physiological role in promotion of pig growth, foetus development, adipose tissue accretion and metabolism, and other physiological functions. However, there are still many physiological functions of GH and GHR that have not been discovered in pigs, therefore, porcine a GHR-specific antagonist serves as a useful tool to further explore the functions of the GH-GHR system, and AN98 has potential in pGH studies.

In summary, in this study, we screened and characterized a novel neutralizing monoclonal anti-pGHR antibody (denoted by AN98). We found that AN98 effectively antagonizes GHR-mediated signaling in vitro. In addition, AN98 shares common GHR-binding epitopes with pGH; therefore, AN98 could inhibit pGH binding to pGHR. The current findings suggest that AN98 is as a pGHRspecific antagonist, which is a useful tool for pGHR-related research in domestic pig.

\section{CONFLICT OF INTEREST}

We certify that there is no conflict of interest with any financial organization regarding the material discussed in the manuscript.

\section{ACKNOWLEDGMENTS}

This work was supported by the Natural Science Foundation Project of Jilin Provincial Science and 
Technology Department (Grant No.20150101107JC, 20140101028JC).

\section{REFERENCES}

Abdel-Meguid, S. S., H. S. Shieh, W. W. Smith, H. E. Dayriner, B. N. Violand, and L. A. Bentle. 1987. Three dimensional structure of a genetically engineered variant of porcine growth hormone. Proc. Natl. Acad. Sci. USA. 84:6434-6437.

Barnard, R., P. G. Bundesen, D. B. Rylatt, and M. J. Waters. 1984. Monoclonal antibodies to the rabbit liver growth hormone receptor: Production and characterization. Endocrinology 115:1805-1813.

Brooks, A. J. and M. J. Waters. 2010. The growth hormone receptor: mechanism of activation and clinical implications. Nat. Rev. Endocrinol. 6:515-525.

Brooks, A. J., W. Dai, M. L. O’Mara, D. Abankwa, Y. Chhabra, R. A. Pelekanos, O. Gardon, K. A. Tunny, K. M. Blucher, and C. J. Morton et al. 2014. Mechanism of activation of protein kinase JAK2 by the growth hormone receptor. Science 344:1249783.

Chen, W. Y., D. C. Wight, B. V. Mehta, T. E. Wagner, and J. J. Kopchick. 1991. Glycine 119 of bovine growth hormone is critical for growth-promoting activity. Mol. Endocrinol. 5:1845-1852.

Chen, W. Y., N. Y. Chen, J. Yun, T. E. Wagner, and J. J. Kopchick. 1994. In vitro and in vivo studies of antagonistic effects of human growth hormone analogs. J. Biol. Chem. 269:1589215897.

Chung, C. S., T. D. Etherton, and J. P. Wiggins. 1985. Stimulation of swine growth by porcine growth hormone. J. Anim. Sci. 60:118-130. de Vos, A. M., M. Ultsch, and A. A. Kossiakoff. 1992. Human growth hormone and extracellular domain of its receptor: crystal structure of the complex. Science 255:306-312.

Gatford, K. L., J. M. Boyce, K. Blackmore, R. J. Smits, R. G. Campbell, and P. C. Owens. 2004. Long-term, but not shortterm, treatment with somatotropin during pregnancy in underfed pigs increases the body size of progeny at birth. J. Anim. Sci. 82:93-101.

Lan, H. N., H. L. Jiang, W. Li, T. C. Wu, P. Hong, Y. M. Li, H. Zhang, H. Z. Cui, and X. Zheng. 2015a. Development and characterization of a novel anti-idiotypic monoclonal antibody to growth hormone, which can mimic physiological functions of growth hormone in primary porcine hepatocytes. Asian Australas. J. Anim. Sci. 28:573-583.

Lan, H. N., W. Li, Z. Fu, Y. Yang, T. Wu, Y. Liu, H. Zhang, H. Cui, Y. Li, P. Hong, J. Liu, and X. Zheng. 2014. Differential intracellular signalling properties of the growth hormone receptor induced by the activation of an anti-GHR antibody. Mol. Cell. Endocrinol. 390:54-64.

Lan, H. N., X. Zheng, M. A. Khan, and S. Li. 2015b. Antiidiotypic antibody: A new strategy for the development of a growth hormone receptor antagonist. Int. J. Biochem. Cell. Biol. 68:101-108.

Ramsay, T. G. and M. P. Richards. 2005. Leptin and leptin receptor expression in skeletal muscle and adipose tissue in response to in vivo porcine somatotropin treatment. J. Anim. Sci. 83:25012508.

Sun, F., Y. Liu, H. Sun, and B. Tian. 2015. Development and characterization of a novel GHR antibody antagonist, GF185. Int. J. Biol. Macromol. 79:864-870.

Van der Lely, A. J., A. F. Muller, J. A. Janssen, R. J. Davis, K. A. Zib, J. A. Scarlett, and S. W. Lamberts. 2001. Control of tumor size and disease activity during cotreatment with octreotide and the growth hormone receptor antagonist pegvisomant in an acromegalic patient. J. Clin. Endocrinol. Metab. 86:478-481. 